\title{
REPRESENTATION AND BOUNDS FOR THE GENERAL FRACTIONAL FACTORIAL.
}

\author{
D. A. Anderson
}

Department of Statistics, University of Wyoming, Laramie, WY 82071, U.S.A.

W. T. Federer

Biometrics Unit, Cornell University, Ithaca, NY 14853, U.S.A.

BU-1209-MA

August, 1994

Key words and phrases: D-optimal; D-minimal; main effect design; Hadamard bound; main effect and interaction design; symmetrical and asymmetrical factorials.

AMS Subject Classification:

ABSTRACT

Let $\mathbf{T}$ denote a main effect plan for the $\prod s_{i}=s_{1} \times s_{2} \quad \ldots \times s_{n}$ factorial with $N$

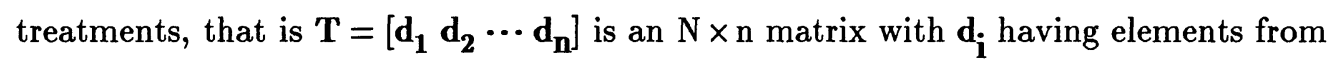
the $\left\{0,1, \cdots, s_{i}-1\right\}$. Denote by $\mathbf{T}_{1}, \mathbf{T}_{2}, \cdots, \mathbf{T}_{\mathbf{n}}$ the $\mathrm{N} \times \mathrm{s}_{\mathrm{i}}$ matrices of indicator variables of $\mathbf{d}_{\mathbf{1}}, \mathbf{d}_{\mathbf{2}}, \cdots, \mathbf{d}_{\mathbf{n}}$, respectively. That is $\mathbf{T}_{\mathbf{i}}=\left[\mathbf{d}_{\mathbf{i}}(0) \mathbf{d}_{\mathbf{i}}(1) \cdots \mathbf{d}_{\mathbf{i}}\left(\mathbf{s}_{\mathbf{i}}-1\right)\right]$ where $\sum_{j=0}^{s_{i}-1} d_{i}(j)=1$ and $\mathbf{d}_{i}=\sum_{j=0}^{s_{i}-1} j d_{i}(j)$. In the usual way we write $E\{Y\}=X \boldsymbol{\alpha}$, where $\mathbf{X}$ is a $(0,1)$ design matrix corresponding to $\mathbf{T}$. A transformation $\mathbf{G}$ is obtained for which $X=X^{*} G^{\prime}$, where $X^{*}=\left[\begin{array}{l}1 \\ 1\end{array} T_{1}^{*} \vdots \cdots \vdots T_{n}^{*}\right]$ thus giving a representation of the design matrix directly in terms of a full rank $(0,1)$-incidence matrix. The determinants of $\mathbf{X}^{\prime} \mathbf{X}, \mathbf{X}^{* \prime} \mathbf{X}^{*}$, and $\mathbf{G}^{\prime} \mathbf{G}$ are evaluated. The determinant of the information matrix is directly expressible in terms of the determinant of a $(0,1)$ matrix. Upper and lower bounds are obtained for the determinant values of $\mathbf{X}^{*}$ when $\mathbf{X}^{*}$ is square and in general for $\mathbf{X}^{* \prime} \mathbf{X}^{*}$. One important aspect of this representation is that the construction of main effect plans and an assessment of their goodness via the determinant criteria can be studied directly in terms of $(0,1)$ matrices. These results are extended to include fractional replicates for estimating interactions.

\section{INTRODUCTION}

In a factorial experiment involving $n$ factors with the $i^{\text {th }}$ factor $F_{i}$ at $s_{i}$ levels $\mathrm{i}=1,2, \cdots, \mathrm{n}$, the total number of treatment combinations is $\prod_{\mathrm{i}=1}^{n} \mathrm{~s}_{\mathrm{i}} \cdot \mathrm{A}$ design is 
usually represented as an $\mathrm{N} \times \mathrm{n}$ matrix $\mathbf{T}$ whose $\mathrm{N}$ rows denote the particular treatment combinations and whose $n$ columns correspond to the levels of the $n$ factors. The elements of a column of $\mathbf{T}$ corresponding to a factor at $s_{i}$ levels are integers from $\left\{0,1, \cdots, s_{i}-1\right\}, i=1,2, \cdots, n$, to denote the $s_{i}$ levels.

In the analysis, the matrix $\mathbf{T}$ is often replaced by a matrix $\mathbf{X}_{\mathrm{N} \times \mathbf{v}}$ which reflects the $v=1+\sum\left(s_{i}-1\right)$ single degree of freedom parametric contrasts in the parametric vector $\boldsymbol{\beta}_{\mathrm{v} \times 1}$ from the usual regression equation $\mathrm{E}\{\mathbf{Y}\}=\mathbf{X} \boldsymbol{\beta}$ and $\operatorname{Cov}\{\mathbf{Y}\}=\sigma^{2} \mathbf{I}_{\mathrm{N}}$. The normal equations are $\mathbf{X}^{\prime} \mathbf{X} \hat{\boldsymbol{\beta}}=\mathbf{X}^{\prime} \mathbf{Y}$, and solutions to the normal equations provide best linear unbiased estimates of estimable functions of the parameters in $\boldsymbol{\beta}$. The matrix $\mathbf{X}^{\prime} \mathbf{X}$ is called the information matrix of the design $\mathbf{T}$, and if $\mathbf{X}^{\prime} \mathbf{X}$ is nonsingular the variance-covariance matrix of $\boldsymbol{\beta}$ is proportional to $\left(\mathbf{X}^{\prime} \mathbf{X}\right)^{-1}$. Most criteria of goodness of a design depend upon some function of $\left(\mathbf{X}^{\prime} \mathbf{X}\right)^{-1}$ as, for example, the determinant, trace, and maximum root criteria. If $\mathbf{X}^{\prime} \mathbf{X}$ is singular we consider a conditional inverse $\left(\mathbf{X}^{\prime} \mathbf{X}\right)^{-}$and restrict to estimable functions of elements of $\boldsymbol{\beta}$.

Raktoe and Federer [1970] obtained such a representation directly in terms of the $(0,1)$ matrix $(1: T)$ for main effect plans for the $2^{\mathrm{n}}$ factorial, where 1 denotes a vector with every element unity. In Section 2 of this paper we present a similar representation for the $\prod_{i=1}^{n} s_{i}$ factorial, and we represent $\left|X^{* \prime} X^{*}\right|$ directly in terms of this representation, where $\mathbf{X}=\mathbf{X}^{*} \mathbf{G}^{\prime}$. In the third section an upper bound on the $\left|\mathbf{X}^{*}\right|$ is obtained for both the symmetric and asymmetric factorials, and the minimum nonzero value of this determinant is indicated. The importance of the representation presented lies in the insight that may be gained toward the construction of fractional factorial plans and the assessment of their goodness via the determinant criteria. In Section 5 , the results are extended to include interactions.

\section{REPRESENTATION OF MAIN EFFECT PLANS IN TERMS OF $(0,1)$ MATRICES}

Consider the general asymmetrical factorial with $i^{\text {th }}$ factor $F_{i}$ at $s_{i}$ levels which is denoted by $s_{1} \times s_{2} \times \cdots \times s_{n}=\prod_{i=1}^{n} s_{i}$. Let $\mathbf{T}$ be an $\mathrm{N} \times \mathrm{n}$ matrix whose rows are a collection of $\mathrm{N}$ treatment combinations (not necessarily distinct) from the IIs $\mathrm{i}$ factorial. Denote by $\mathbf{d}_{i}$ the $i^{\text {th }}$ column of $\mathbf{T}$ corresponding to the $i^{\text {th }}$ factor so that $\mathbf{T}=\left[\begin{array}{lll}\mathbf{d}_{\mathbf{1}} \mathbf{d}_{\mathbf{2}} \cdots \mathbf{d}_{\mathbf{n}}\end{array}\right]$. Since the $\mathrm{i}^{\text {th }}$ factor has $\mathrm{s}_{\mathbf{i}}$ levels denoted by $0,1, \cdots, \mathrm{s}_{\mathbf{i}}-1$, the 
column $\mathbf{d}_{\mathbf{i}}$ contains these $s_{\mathbf{i}}$ symbols. Next let $\mathbf{T}_{\mathbf{i}}=\left[\mathbf{d}_{\mathbf{i}}(0) \mathbf{d}_{\mathbf{i}}(1) \cdots \mathbf{d}_{\mathbf{i}}\left(\mathrm{s}_{\mathbf{i}}-1\right)\right]$ be an $\mathrm{N} \times \mathrm{s}_{\mathrm{i}}$ matrix where the $\mathrm{j}^{\text {th }}$ column is the indicator variable for level $\mathrm{j}$ of the $\mathrm{i}^{\text {th }}$ factor. For each i

$$
\sum_{j=0}^{s_{i}^{-1}} d_{i}(j)=1 \quad \text { and } \quad d_{i}=\sum_{j=0}^{s_{i}^{-1}} j d_{i}(j)
$$

where 1 is a column vector of all ones. The usual over-parameterized model can be expressed as

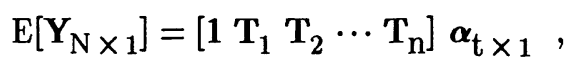

where $\boldsymbol{\alpha}^{\prime}=\left[\mu, \alpha_{10} \alpha_{11} \cdots \alpha_{1 \mathrm{~s}_{\mathrm{i}}-1} \cdots \alpha_{\mathrm{n} 0} \alpha_{\mathrm{n} 1} \cdots \alpha_{\mathrm{ns}_{\mathrm{n}}-1}\right]$ and $\mathrm{t}=1+\sum_{\mathrm{i}=1}^{\mathrm{n}} \mathrm{s}_{\mathrm{i}}$. If $\mathbf{T}$ is a connected main effect plan, the rank of $\left[\begin{array}{lllll}1 & T_{1} & T_{2} & \cdots & T_{n}\end{array}\right]$ is $t-n . ~ i=1$ order to obtain unique estimates of estimable contrasts of the parameters it is common to introduce $\mathrm{n}$ constraints. One choice is $\alpha_{\mathrm{i} 0}=0$ for $\mathrm{i}=1,2, \cdots, \mathrm{n}$. These constraints result in the matrix

$$
\mathbf{X}_{\mathrm{N} \times \mathrm{v}}^{*}=\left[\begin{array}{llll}
1 & \mathbf{T}_{1}^{*} & \mathbf{T}_{\mathbf{2}}^{*} \cdots \mathbf{T}_{\mathbf{n}}^{*}
\end{array}\right],
$$

where $T_{i}^{*}$ is obtained from $T_{\mathbf{i}}$ by deleting the first column of $\mathbf{T}_{\mathbf{i}}$ and $\mathrm{v}=1+\sum_{\mathrm{i}=1}^{\mathrm{n}}\left(\mathrm{s}_{\mathrm{i}}-1\right)=\mathrm{t}-\mathrm{n}$. This $(0,1)$ matrix $\mathrm{X}^{*}$ has full column rank. In this form we are able to utilize the theory and properties of $(0,1)$ matrices, and results such as obtained, for example, by Raktoe and Federer (1970) and Anderson and Federer (1975).

Typically main effects are defined in terms of some set of (normalized) orthogonal contrasts, Raktoe et al. (1981). It is well-known that any two sets of orthogonal contrasts are related by an orthogonal transform. Hence it is sufficient to consider any one. Any other set of non-orthogonal (but full rank) contrasts can be obtained from an orthogonal set by a non-orthogonal transform. For example, the contrasts $\alpha_{i j}-\alpha_{i 0}, \mathrm{j}=1,2, \cdots, \mathrm{s}_{\mathrm{i}}-1, \mathrm{i}=1,2, \cdots, \mathrm{n}$ are a common (treatment minus control) set of non-orthogonal contrasts. For convenience we shall use the normalized Helmert orthogonal contrasts on $s_{i}$ symbols. These are

$$
\left[\begin{array}{c}
\mathbf{1}^{\prime} / \sqrt{\mathbf{s}_{\mathbf{i}}} \\
\mathbf{C}_{\mathbf{i}}
\end{array}\right]=\boldsymbol{\Delta}_{\mathrm{i}}^{-1}\left[\begin{array}{ccccc}
1 & 1 & 1 & \cdots & 1 \\
1 & -1 & 0 & \cdots & 0 \\
1 & 1 & -2 & \cdots & 0 \\
1 & 1 & 1 & \cdots & \mathrm{s}_{\mathrm{i}}-1
\end{array}\right]
$$


where $\Delta_{i}=\operatorname{Diag}\left[\mathrm{s}_{\mathrm{i}} 1 \cdot 2 \quad 2 \cdot 3 \quad \cdots\left(\mathrm{s}_{\mathrm{i}}-1\right)\left(\mathrm{s}_{\mathrm{i}}\right)\right]$ is the $\mathrm{s}_{\mathrm{i}} \times \mathrm{s}_{\mathrm{i}}$ matrix with row sums of squares on the diagonal. Clearly this is an orthogonal matrix, and hence its determinant is one. Then if $\mathbf{Y}_{\mathrm{N} \times 1}$ is again the $\mathrm{N} \times 1$ vector of observations corresponding to $\mathbf{T}$, in this parameterization we have

$$
\mathrm{E}\left\{\mathbf{Y}_{\mathrm{N}}\right\}=\mathbf{X} \boldsymbol{\beta},
$$

where $\beta=\left(\begin{array}{llllllllll}\mu & \beta_{11} & \beta_{12} & \cdots & \beta_{1\left(\mathrm{~s}_{1}-1\right)} & \cdots & \beta_{\mathrm{n} 1} & \beta_{\mathrm{n} 2} & \cdots & \beta_{\mathrm{n}\left(\mathrm{s}_{\mathrm{n}}-1\right)}\end{array}\right)^{\prime}$ denotes the $\mathrm{v}=1+\sum_{\mathrm{i}=1}^{\mathrm{n}}\left(\mathrm{s}_{\mathrm{i}}-1\right)$ vector of single degree of freedom contrasts derived from (2.4), and

$$
X=\left[\begin{array}{llllllll}
1 & T_{1} & C_{1}^{\prime} & T_{2} & C_{2}^{\prime} & \cdots & T_{n} & C_{n}^{\prime}
\end{array}\right]
$$

The objective in this section is to establish the relationship between the $(0,1)$ matrix $\mathrm{X}^{*}$ of (2.3) with $\mathrm{X}$ of (2.6) which will permit comparison of determinant criteria of competing designs. To this end, let

$\mathbf{G}_{\mathbf{i}}=\left[\begin{array}{cc}1 & \mathbf{1}^{\prime} / \mathrm{s}_{\mathbf{i}} \\ \mathbf{0} & \mathbf{H}_{\mathbf{i}}\end{array}\right]=\left[\begin{array}{ccccc}1 & 1 / \mathrm{s}_{\mathrm{i}} & 1 / \mathrm{s}_{\mathrm{i}} & \cdots & 1 / \mathrm{s}_{\mathrm{i}} \\ 0 & -\sqrt{2} / 2 & 0 & \cdots & 0 \\ 0 & \frac{1}{\sqrt{2 \cdot 3}} & -\sqrt{2 \cdot 3} / 3 & \cdots & 0 \\ \vdots & & & & \\ 0 & \frac{1}{\sqrt{\mathrm{s}_{\mathrm{i}}\left(\mathrm{s}_{\mathrm{i}}-1\right)}} & \frac{1}{\sqrt{\mathrm{s}_{\mathrm{i}}\left(\mathrm{s}_{\mathrm{i}}-1\right)}} & \cdots & -\sqrt{\mathrm{s}_{\mathrm{i}}\left(\mathrm{s}_{\mathrm{i}}-1\right)} / \mathrm{s}_{\mathrm{i}}\end{array}\right]$,

and for all $\mathrm{n}$ factors

$$
\mathbf{G}=\left[\begin{array}{ccccc}
1 & \mathbf{1}^{\prime} / \mathrm{s}_{1} & \mathbf{1}^{\prime} / \mathrm{s}_{2} & \ldots & \mathbf{1}^{\prime} / \mathrm{s}_{\mathrm{n}} \\
\mathbf{0} & \mathbf{H}_{1} & \mathbf{0} & \cdots & \mathbf{0} \\
\mathbf{0} & \mathbf{0} & \mathbf{H}_{2} & \cdots & \mathbf{0} \\
\vdots & & & & \\
\mathbf{0} & \mathbf{0} & \mathbf{0} & \cdots & \mathbf{H}_{\mathrm{n}}
\end{array}\right]
$$

It can be ascertained by direct calculation that if $\mathbf{X}^{*}$ and $\mathbf{X}$ are defined by (2.3) and 
(2.6) respectively, the following relationships hold:

Theorem 2.1 For the general asymmetric factorial

(a) $\mathbf{X G}=\mathrm{X}^{*}=\left[\begin{array}{lllll}1 & \mathrm{~T}_{1}^{*} & \mathrm{~T}_{2}^{*} & \ldots & \mathrm{T}_{\mathrm{n}}^{*}\end{array}\right]$,

(b) $|\mathbf{G}|=\prod_{\mathrm{i}=1}^{\mathrm{n}}\left(\mathrm{s}_{\mathrm{i}}\right)^{-\frac{1}{2}}$,

(c) $\left|\mathbf{X}^{\prime} \mathbf{X}\right|=\prod_{\mathrm{i}=1}^{\mathrm{n}}\left(\mathrm{s}_{\mathrm{i}}\right) \quad\left|\mathbf{X}^{* \prime} \mathbf{X}^{*}\right|$, and

(d) if $\mathbf{T}$ is saturated, $\mathrm{N}=\mathrm{v},|\mathbf{X}|=\prod_{\mathrm{i}=1}^{\mathrm{n}}\left(\mathrm{s}_{\mathrm{i}}\right)^{\frac{1}{2}}\left|\mathbf{X}^{*}\right|$.

Corollary 2.1 For the $\mathrm{s}^{\mathrm{n}}$ symmetrical factorial

(a) $|\mathrm{G}|=\mathrm{s}^{-\frac{\mathrm{n}}{2}}$,

(b) $\left|\mathbf{X}^{\prime} \mathbf{X}\right|=\mathrm{s}^{\mathrm{n}}\left|\mathbf{X}^{* \prime} \mathbf{X}^{*}\right|$, and

(c) if $\mathbf{T}$ is saturated, $|\mathbf{X}|=\mathrm{s}^{\frac{\mathrm{n}}{2}}\left|\mathbf{X}^{*}\right|$.

Thus the determinant of $\mathbf{X}^{\prime} \mathbf{X}$ for any normalized orthogonal contrast definition of effects is a multiple $\prod_{i=1}^{n}\left(s_{i}\right)$ of the determinant $X^{* \prime} X^{*}$ from the corresponding $(0,1)$ matrix.

\section{BOUNDS ON THE DETERMINANTS OF NON-SINGULAR DESIGN MATRICES}

The transformation from $\mathbf{X}$ to $\mathbf{X}^{*}$ provides a simple proof that the determinant of $\mathbf{X}^{\prime} \mathbf{X}$ for main effect plans is invariant to any change of level designation for any factor. Any permutation of the non-zero levels results only in a corresponding permutation of columns in $\mathbf{X}^{*}$, which of course does not change the value of the determinant of $\mathbf{X}^{* \prime} \mathbf{X}^{*}$. Likewise, any non-zero level may be interchanged with the zero level for any specified factor. The corresponding change in $\mathbf{X}^{*}$ is a linear combination of the first column of all ones and the columns of $\mathbf{T}_{\mathbf{1}}, \mathbf{T}_{\mathbf{2}}, \cdots \mathbf{T}_{\mathbf{n}}$ corresponding to that factor. Again, this does not change the determinant. This invariance property is a well known result (see, for example, Paik and Federer [1970], Joiner [1973], and Srivastava, Raktoe, and Pesotan [1976]). The representation in 
terms of $(0,1)$ matrices makes it more apparent and provides a simpler approach to their results.

Let $\lambda_{\mathrm{i}}^{\mathrm{k}}, \mathrm{k}=0,1,2, \cdots, \mathrm{s}_{\mathrm{i}}-1$ denote the number of treatment combinations of a fraction $\mathbf{T}$ which contain the $\mathrm{i}^{\text {th }}$ factor at level $\mathrm{k}$. Then $\sum_{\mathrm{k}=0}^{\mathrm{s}-1} \lambda_{\mathrm{i}}^{\mathrm{k}}=\mathrm{N}$ for each $\mathrm{i}$. In any discussion involving the determinant of $\mathbf{X}$, or of $\mathbf{X}^{*}$, we may, without loss of generality, assume that $\lambda_{i}^{0} \geq \lambda_{1}^{1} \geq \lambda_{1}^{2} \geq \cdots \geq \lambda_{i}^{s-1}$ for each $\mathrm{i}$ because of the invariance property. Raktoe and Federer [1970] obtained the following bound on $\left\|\mathbf{X}^{*}\right\|$ using Hadamard's theorem, when normalized.

$$
\left\|\mathrm{X}^{*}\right\| \leq 2^{-\frac{\mathrm{n}}{2}}(\mathrm{n}+1)^{(\mathrm{n}+1) / 2} \text {. }
$$

Since $\left|\mathbf{X}^{*}\right|$ must be an integer, we take the integer part of the right hand side of (3.1) as the upper bound. We now obtain a generalization of their result for $\mathbf{X}^{*}$ matrices, and consequently $\mathbf{X}$ matrices, for saturated main effect plans from the general

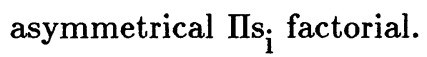

Theorem 3.1 Let $\mathbf{T}$ be a saturated main effect plan for the $\prod_{i=1}^{n} s_{i}$ factorial with $\mathrm{N}=\sum_{\mathrm{i}=1}^{\mathrm{n}}\left(\mathrm{s}_{\mathbf{i}}-1\right)+1$. If $\mathbf{X}^{*}=\left[\mathbf{1}: \mathbf{T}_{\mathbf{1}}^{*}: \mathbf{T}_{\mathbf{2}}^{*}: \ldots: \mathbf{T}_{\mathbf{n}}^{*}\right]$, then

$$
\left|X^{*}\right| \leq \text { integer part of } N^{\frac{N}{2}} \prod_{i=1}^{n} s_{i}-s_{i} / 2
$$

For the $s^{n}$ symmetric factorial this reduces to $\left|X^{*}\right| \leq$ integer part of $N^{\frac{N}{2}} s^{-n s / 2}$. When $\mathrm{s}=2$, this reduces to equation (3.1).

Proof: From Theorem 2.1,

$$
\left\|\mathbf{X}^{*}\right\|=\prod_{\mathrm{i}=1}^{\mathrm{n}} \mathrm{s}_{\mathrm{i}}^{-\frac{1}{2}}\|\mathbf{X}\|=\prod_{\mathrm{i}=1}^{\mathrm{n}} \mathrm{s}_{\mathrm{i}}^{-\frac{1}{2}}\left|\mathbf{X}^{\prime} \mathbf{X}\right|^{\frac{1}{2}}
$$

Then the proof follows from Hadamards's determinant theorem.

Corollary 3.1 Let $\mathrm{T}$ be a main effect plan for a $\Pi \mathrm{s}_{\mathrm{i}}$ factorial experiment with $\mathrm{N} \geq$ $\mathrm{v}=1+\sum_{\mathrm{i}=1}^{\mathrm{n}}(\mathrm{s}-1)$. Then

$$
\begin{aligned}
\left|\mathbf{X}^{* /} \mathbf{X}^{*}\right| \leq & \text { integer part of } \mathrm{N}^{\mathrm{v}} \Pi \mathrm{s}_{\mathrm{i}}^{-\mathrm{s}_{\mathrm{i}}} \text {, asymmetrical factorial and } \\
& \leq \text { integer part of } \mathrm{N}^{\mathrm{v}} \mathrm{s}^{-\mathrm{ns}}, \text { symmetrical factorial. }
\end{aligned}
$$

Proof: The proof of theorem 3.1 uses $\left|\mathbf{X}^{\prime} \mathbf{X}\right|$ and the essential steps do not depend on 
$\mathrm{N}=\sum_{\mathrm{i}=1}^{\mathrm{n}}\left(\mathrm{s}_{\mathrm{i}}-1\right)+1 . \quad$ Hence the proof is complete.

Theorem 3.2 The class of saturated main effect designs for the $\prod_{i=1}^{n} s_{i}$ factorial contains designs for which $\left|\mathbf{X}^{*}\right|=1$. That is, the minimum possible non-zero value is always attainable.

Proof: The familiar "one at a time design" has a $(0,1)$ representation as

$$
\mathbf{X}^{*}=\left[\begin{array}{ccc}
1 & 0 & 0 \ldots 0 \\
\underline{1} & \mathbf{I}_{\mathrm{v}-1}
\end{array}\right], \mathrm{v}-1=\sum_{\mathrm{i}=1}^{\mathrm{n}}\left(\mathrm{s}_{\mathrm{i}}-1\right)
$$

whose determinant is clearly one. The proof is complete since one design is exhibited for every case.

Corollary 3.2 If $\mathbf{T}$ is a saturated main effect plan for the $\prod_{\mathrm{i}=1}^{\mathrm{n}} \mathrm{s}_{\mathrm{i}}$ factorial, the minimum possible value of $\left|\mathbf{X}^{\prime} \mathbf{X}\right|$ is $\prod_{i=1}^{n} s_{i}$ and this value is always attainable. Thus for any saturated design, $\left|\mathbf{X}^{\prime} \mathbf{X}\right|$ is a multiple of this minimum value.

Proof: This follows directly from theorems 2.1 and 3.2. It is noted that the spectrum of values for $\left\|\mathbf{X}_{(\mathrm{n}+1) \times(n+1)}^{*}\right\|$ contains all values attainable for $\left\|\mathbf{X}_{\mathrm{n} \times \mathrm{n}}^{*}\right\|$. This is easily demonstrated by constructing an

$$
\mathbf{X}_{(\mathrm{n}+1) \times(\mathrm{n}+1)}^{*}=\left(\begin{array}{ll}
\mathbf{1}_{(\mathrm{n}+1) \times 1} & \vdots \\
& \mathbf{0}_{1 \times \mathrm{n}}^{\prime} \\
& \mathbf{X}_{\mathrm{n} \times \mathrm{n}}^{*}
\end{array}\right) \text {, the determinant (absolute value) of }
$$

which has the same spectrum of values as $\left\|\mathbf{X}_{\mathrm{n} \times \mathrm{n}}^{*}\right\|$. This means that a lower bound on the upper bound of $\left\|X_{(n+1) \times(n+1)}^{*}\right\|$ can be obtained from the maximum value of $\left\|\mathbf{X}_{n \times n}^{*}\right\|$. In most cases, this latter value is unknown. For such situations, use the maximum value of $\left\|\mathrm{X}_{\mathrm{p} \times \mathrm{p}}^{*}\right\|, \mathrm{p} \leq \mathrm{n}$, for the $\mathrm{p}$ for which it is known, e.g., for an orthogonal array. Then, any design $\mathbf{T}$ which does not have an $\left\|\mathbf{X}_{(\mathrm{n}+1) \times(\mathrm{n}+1)}\right\|$ between the lower bound on the upper bound and the upper bound is not a good design with respect to D-optimality. A design $\mathbf{T}$ with an

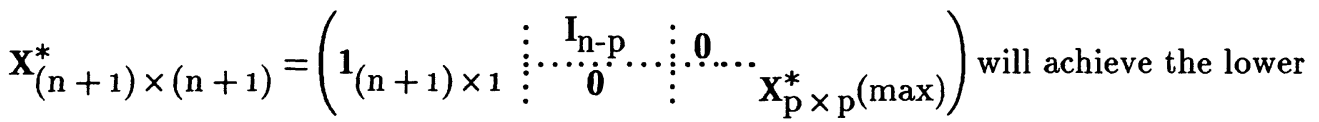
bound on the upper bound. 
Theorem 3.3 A necessary and sufficient condition for achieving the upper bound of theorem 3.1 is that

i) all levels of a factor occur with equal frequency, and

ii) all possible pairs of levels of two factors appear with the same frequency.

Proof: (Necessary) Part i) has already been shown in theorem 3.1. For a pair of factors with $s_{i}$ and $s_{j}$ levels, respectively, there are $\left(s_{i}-1\right)\left(s_{j}-1\right)$ inner products which must be zero. There are $\left(s_{i}-1\right)\left(s_{j}-1\right)$ equations relating the equal frequencies of each level, and one equation relating to total number of runs. Thus there are $1+\left(s_{i}-1\right)+\left(s_{j}-1\right)+\left(s_{i}-1\right)\left(s_{j}-1\right)=s_{i} s_{j}$ equations (of full rank) involving the number of times each pair of levels appears. The unique solution is $\mathrm{N} / \mathrm{s}_{\mathrm{i}} \mathrm{s}_{\mathrm{j}}$ for each pair. The sufficiency is trivial.

\section{ON THE CONSTRUCTION OF MAIN EFFECT PLANS}

Anderson and Federer (1975) use ten methods to construct fractional factorials and mention others. Raktoe (1981) et al. discuss more than 20 methods for constructing fractional factorials. A recent paper by Cheng et al. (1993) presents a catalogue of fractional replicate designs for $16,27,32$, and 64 runs. Despite all these methods, there is no procedure for constructing general D-optimal fractions from the general asymmetrical factorial. Bounds given by theorems 3.1 and 3.2 are useful for comparison of any given fractional replicate. Federer and Pal (1980) present a procedure for constructing saturated main effect plans for $k\left(p^{t}\right)$ factorials. One of the factors at $p$ levels is in a balanced, bibd, or partially balanced, pbibd, arrangement with the other $t-1$ factors at $p$ levels. The factor at $k$ levels is orthogonal to all $t$ factors at $p$ levels and the $t-1$ factors which are in a bibd or pbibd arrangement with one of the factors at $p$ levels are in a bibd or pbibd with each other. The number $t$ is determined by the number of pairwise mutually orthogonal latin squares in the set MOL $(p, t)$. After super-imposing the squares one on the other the k-rows of the set are arranged in such a manner that the factor corresponding to the columns is in a bibd arrangement with $\lambda$ occurrences of pairs of levels on in a pbibd arrangement with $\lambda$ or $\lambda+1$ occurences of pairs of levels with each one of the $t-1$ factors at $\mathrm{p}$ levels corresponding to the treatments of the MOL set. Note that $2 \leq \mathrm{k}$ and that $\mathrm{k}$ may be greater than $\mathrm{p}$ by duplicating the rows of the MOL set. Although 
these fractions do not achieve the upper bound in Theorem 3.1, there is no assurance that better fractional replicates can be obtained. With these properties the construction of main effect plans for the symmetric and asymmetric factorial may now be directly related to constructions of $(0,1)$ matrices with certain constraints on the columns. Thus the body of knowledge and developed theory of $(0,1)$ matrices can be directly brought to the construction of main effect plans.

\section{EXTENTION TO INCLUDE INTERACTION TERMS}

If $\mathrm{s}$ is a prime or prime power, it is possible to include interactions in the $(0$, 1) representation of the $s^{n}$ factorial. This representation is in terms of the geometric definition of the factorial effects. In this definition of factorial effects $F_{i} F_{j}^{a}$ $a \neq 0 \in \mathrm{GF}(\mathrm{s})$ is used to denote $(\mathrm{s}-1)$ degrees of freedom belonging to the interaction of the $\mathrm{i}^{\text {th }}$ and $\mathrm{j}^{\text {th }}$ factors. As a ranges over the $\mathrm{s}-1$ non-zero values of the Galois field of order $s, G F(s)$, all $(s-1)(s-1)$ degrees of freedom for the interaction between $F_{i}$ and $F_{j}$ are identified. A general $k^{\text {th }}$ order component with $(s-1)$ degrees of freedom is denoted by $\mathrm{F}_{\mathrm{i}_{1}} \mathrm{~F}_{\mathrm{i}_{2}}^{\mathrm{a}_{2}} \ldots \mathrm{F}_{\mathrm{i}_{\mathrm{k}}}^{\mathrm{a}_{\mathrm{k}}}$ where $\mathrm{a}_{2}, \mathrm{a}_{3}, \cdots, \mathrm{a}_{\mathrm{k}}$ are nonzero elements of GF(s). As the $a_{2}, a_{3}, \cdots, a_{k}$ range over all possible non-zero values, all $(s-1)^{k-1}(s-1)$ degrees of freedom associated with this $k^{\text {th }}$ order interaction are identified.

If $\mathbf{T}$ denotes a design for the $\mathrm{s}^{\mathrm{n}}$ factorial, denote the columns of $\mathbf{T}$ as $\mathbf{T}=\left[\mathbf{d}_{\mathbf{1}}\right.$ $\mathbf{d}_{\mathbf{2}} \cdots \mathbf{d}_{\mathbf{n}}$ ] . To include the interaction between factors $F_{i}$ and $F_{j}$ in the model, adjoin to $\mathbf{T}$ the $(\mathrm{s}-1)$ columns

$$
\mathbf{d}_{\mathbf{i}}+\mathbf{a} \mathbf{d}_{\mathbf{j}} \quad \mathbf{a} \neq 0 \in \mathrm{GF}(\mathrm{s})
$$

where all calculations are in the field GF(s). Each of these columns clearly contains only the elements of GF(s) and hence have the same form as the columns of T. For higher order interactions, say $\mathrm{F}_{\mathrm{i}_{1}} \mathrm{~F}_{\mathrm{i}_{2}}^{\mathrm{a}_{2}} \cdots \mathrm{F}_{\mathrm{i}_{\mathrm{k}}}^{\mathrm{a}_{\mathrm{k}}}$, we adjoin to $\mathbf{T}$ columns of the form

$$
d_{i_{1}}+a_{i_{2}} d_{i_{2}}+\cdots+a_{i_{k}} d_{i_{k}} \quad a_{i_{1}}, a_{i_{2}}, \cdots a_{i_{k}} \neq 0 \in G F(s)
$$

Let $\mathbf{D}$ denote the $\mathrm{N} \times(\mathrm{n}+\mathrm{m})$ matrix with $\mathrm{m}$ columns adjoined to $\mathbf{T}$ for all desired interactions. The matrix $\mathbf{D}$ has elements from $G F(s)$ and as in (2.1), we let $\mathbf{D}_{\mathbf{i}}$ denote the $N \times(n+m)$ incidence matrix of $i$ in $D, i \in G F(s)$. Then

$$
\sum_{\mathrm{i} \in \mathrm{GF}(\mathrm{s})} \mathbf{D}_{\mathrm{i}}=\mathbf{J}_{\mathrm{N} \times(\mathrm{n}+\mathrm{m})} \quad \text { and } \quad \mathbf{D}_{\mathrm{i} \in \mathrm{GF}(\mathrm{s})} \mathrm{iD}_{\mathrm{i}} .
$$


The $\mathbf{X}$ matrix for the model containing interaction terms has the same form as (2.3), that is

$$
X=\left[1: D_{0}-D_{1} \vdots D_{0}+D_{1}-2 D_{2} \vdots \cdots: \sum_{i=0}^{s-2} D_{i}-(s-1) D_{s-1}\right]
$$

It is now apparent that with $\mathrm{v}=1+(\mathrm{n}+\mathrm{m})(\mathrm{s}-1)$, the $\mathrm{v} \times \mathrm{v}$ matrix $\mathbf{G}$ of equation (2.5) may be multiplied by $\mathrm{X}_{\mathrm{N} \times(\mathrm{n}+\mathrm{m})}$ exactly as in (2.4) to produce a $(0,1)$ representation of $\mathbf{X}$. This observation is explicitly stated in Theorem 5.1.

Theorem 5.1 With $\mathbf{X}$ as in (5.4) and the transformations $\mathbf{X G}=\mathbf{X}^{*}$, we have

(a) $\mathrm{X}^{*}=\left[1 \vdots \mathrm{D}_{1} \vdots \mathrm{D}_{2} \vdots \cdots \vdots \mathrm{D}_{\mathrm{s}-1}\right]$

(b) $|\mathbf{G}|=(\mathrm{s} !)^{-(\mathrm{n}+\mathrm{m})}(-1)^{(\mathrm{n}+\mathrm{m})(\mathrm{s}-1)}$,

(c) $|\mathbf{X}|=\mathbf{X}^{*} \mathbf{G}^{-1}$, and

(d) $\quad\left|\mathbf{X}^{\prime} \mathbf{X}\right|=(\mathrm{s} !)^{2(\mathrm{n}+\mathrm{m})}\left|\mathrm{X}^{* \prime} \mathbf{X}^{*}\right|$.

Proof: The theorem follows directly from theorem 2.1.

It should be noted that in the asymmetric factorial $\Pi_{\mathrm{i}}$ that interactions between factors with the same (prime power) number of levels may be included in the model exactly as in the discussion above. For factors with differing numbers of levels or with non-prime power number of levels, the convenient field of order $\mathrm{s}$ does not exist. There may be a corresponding $(0,1)$ representation which includes interaction terms for the general asymmetric factorial relative to some other formulation of the interaction contrasts.

Pesotan and Raktoe [1975] show that the $(0,1)$ representation does not extend in a natural way if the product definition of the effects is used. They do show that such a representation does exist in terms of $(-1,0,1)$ matrices, and exhibit suitable classes of design matrices $\mathbf{T}$ and sets of factorial effects such that a natural $(0,1)$ representation does exist.

\section{ACKNOWLEDGEMENT}

This research was partially supported by NSERC grants No. A8776 and No. A07204. The comments of D. Raghavarao, a referee, and S.R. Searle were helpful and constructive in preparing this version of the paper which is a revision of Anderson and Federer $(1974,1981,1985)$. Illustrative examples are given in these versions but omitted here. 


\section{BIBLIOGRAPHY}

Anderson, D.A. and Federer, W.T (1974). Representation and construction of main effect plans in terms of $(0,1)$ matrices. BU-499-M in the Technical Report Series of the Biometrics Unit, Cornell University, Ithaca, NY.

Anderson, D.A. and Federer, W.T. (1975). Possible absolute determinant values for square $(0,1)$-matrices useful in fractional replication. Utilitas Mathematica 7 , $135-150$.

Anderson, D.A. and Federer, W.T. (1981). Representation and construction of main effect plans in terms of $(0,1)$ matrices. BU-747-M in the Technical Report Series of the Biometrics Unit, Cornell University, Ithaca, NY.

Anderson, D.A. and Federer, W.T. (1985). Zero-one representation and bounds for fractional factorial designs. BU-893-M in the Technical Report Series of the Biometrics Unit, Cornell University, Ithaca, NY.

Federer, W.T. and Pal, B.K. (1980). NA saturated fractional replicate plans. BU709-M in the Technical Report Series of the Biometrics Unit, Cornell University, Ithaca, NY.

Hedayat, A. and Seiden, E. (1970). F-square and orthogonal F-squares design: A generalization of latin squares and orthogonal latin squares design. Ann. Math. Statist. 41, 2035-2044.

Joiner, J.R. (1973). Similarity of designs in fractional factorial experiments. Ph.D. Thesis, Cornell University, Ithaca, NY.

Paik, U.B. and Federer, W.T. (1970). A randomized procedure of saturated main effect fractional replicates. Ann. Math. Statist. 41, 369-375.

Pesotan, H. and Raktoe, B.L. (1975). Remarks on extentions of the AndersonFederer $(0,1)$-matrix procedure for main effects of a higher degree. Comm. in Stat. 4, 797-811.

Raktoe, B.L. and Federer, W.T. (1970). A characterization of optimal saturated main effect plans of the $2^{\mathrm{n}}$ factorial. Ann. Math. Statist. 41, 203-206.

Raktoe, B.L., Hedayat, A., and Federer, W.T. (1981). Factorial Designs, John Wiley \& Sons, Inc., New York.

Srivastava, J.N., Raktoe, B.L., and Pesotan, H. (1976). On invariance and randomization in fractional replication. Ann. Statist. 4, 423-430. 